\title{
Novel Multiple Relay Selection Schemes in Two-Hop Cognitive Relay Networks
}

\author{
Jiali $\mathrm{Xu}^{\dagger}$, Haixia Zhang ${ }^{\dagger}$, Dongfeng Yuan $^{\dagger}$, Qingqing $\mathrm{Jin}^{\dagger}$, Cheng-Xiang Wang ${ }^{\dagger \dagger}$ \\ ${ }^{\dagger}$ School of Information Science and Engineering, Shandong University, Jinan, Shandong, China \\ ${ }^{\dagger \dagger}$ School of Engineering and Physical Sciences, Heriot-Watt University, EH14 4AS, Edinburgh, UK \\ Email: \{xujiali, haixia.zhang, dfyuan, qingqingjin\}@sdu.edu.cn, cheng-xiang.wang@hw.ac.uk
}

\begin{abstract}
This paper studies multiple relay selection schemes in two-hop cognitive relay networks to maximize the signal-tonoise ratio (SNR) of the secondary receiver. We first formulate the relay selection as a nonlinear integral programming, and then propose two suboptimal methods, greedy scheme and hybrid scheme. Based on the sorted efficiency function, the greedy scheme selects the secondary relay nodes provided that the sum of the interference power does not exceed the interference power constraint (IPC) of the primary users. The hybrid scheme is proposed to overcome the performance degradation of the greedy scheme whose efficiency function is not the optimal ordering function. Numerical results indicate that the greedy scheme can sometimes, but not always, achieve the near-optimal SNR performance. The hybrid scheme always outperforms the greedy scheme and any best single scheme. Moreover, both the greedy scheme and hybrid scheme are of low complexity compared to the exhaustive scheme.
\end{abstract}

Keywords-Multiple relay selection; SNR maximization; cognitive relay networks

\section{INTRODUCTION}

Cognitive relay networks have attracted great attention of research in academic and industrial areas, since it jointly considers the cognitive radio (CR) and cooperative relay techniques [1]-[4]. CR networks are gaining prominence with the development of CR technology, which aims at improving the spectral efficiency. Users in CR networks work in two modes, namely overlay and underlay modes. In the overlay mode, secondary users (SUs) can access the spectrum after sensing the spectrum holes, while in the underlay mode, SUs and primary users (PUs) can share the same spectrum band simultaneously providing that the interference power constraint (IPC) of the PUs is satisfied[4][5]. In this paper, we focus on the second mode. Additionally, in cognitive relay networks cooperative relay scheme is employed to combat the multipath fading by increasing transmit diversity gain in wireless networks. Here, we consider amplify-and-forward (AF) cooperation scheme, in which the relay simply amplifies the received signal and then forwards it.

A main challenge in designing cognitive relay networks is how to effectively select the cooperative relay nodes. There exist some work on single and multiple relay selection [6][10]. In [6], four schemes for single and multiple relay

This work was supported by NSFC (No. 60832008), Key Project of Provincial Scientific Foundation of Shandong (No.Z2008G01), and RCUK for the "UK-China Science Bridges: R\&D on (B)4G Wireless Mobile Communications". selection were proposed and the corresponding diversity orders were investigated. In [7], an optimized relay selection method was addressed based on the combinatorial optimization theory. However, these work did not consider CR scenario. In [8], it was assumed that the relay node only cooperated when the primary user in the corresponding network cluster was absent. In [9], the authors proposed an opportunistic sharing scheme and based on the Nash equilibrium and Pareto efficiency to analyze the performance. In [10], the optimal single relay selection and power allocation were studied, but without considering the multiple relay selection. Although some work about power or spectrum allocation like in [11] bore some resemblance to our work in system model, the work addressed fundamentally different issues.

This paper generalizes the multiple relay selection in twohop cognitive relay networks. The optimal multiple relay selection scheme can be determined via exhaustive search over all combinations of relay selection. However, the exhaustive search will result in an exponential $N$ (the total number of relay nodes) computational complexity, which is unacceptable in the case of large number of relay nodes. In order to overcome this drawback, we first propose a greedy scheme based on the efficiency function. Then a hybrid scheme is addressed to approach the optimal performance.

The rest of this paper is organized as follows. In Section II, we describe the system model and formulate the multiple relay selection in two-hop cognitive relay networks. In Section III we first propose greedy scheme and hybrid scheme, then compare their complexity. Numerical results are presented in Section IV for performance evaluation. Conclusions are drawn in Section $\mathrm{V}$.

\section{System Model And Problem Formulation}

\section{A. System Model}

The system model is depicted in Fig. 1. We consider a two-hop cognitive relay network with a secondary source (SS) node and a secondary destination (SD). Assume that there is no direct link from the SS to SD, e.g., due to large physical separation or sever shadowing effects, and thy communicate with the help of $N$ potential secondary relay (SR) nodes, denoted by $\mathrm{SR}_{j}, j=1, \ldots, N$. The whole secondary network is within the communication range of a primary base station (PBS). Each node is equipped with single antenna and each relay works in AF mode. Denote the channel gain from SS to $\mathrm{SR}_{j}$ with $h_{s j}$ and the channel gain from $\mathrm{SR}_{j}$ to SD with $h_{j d}$. 


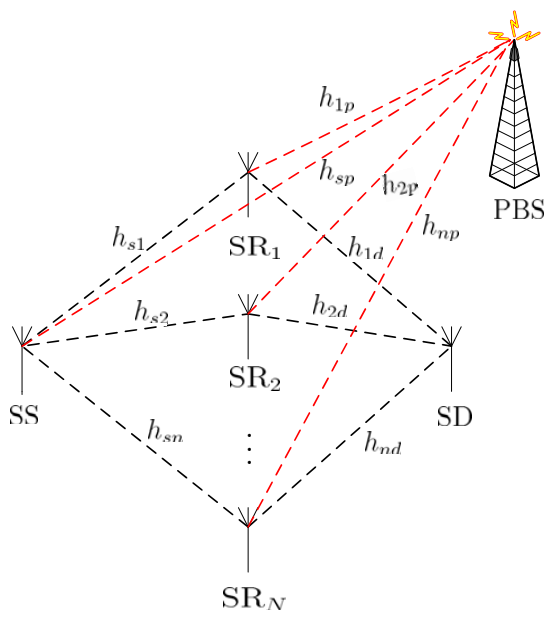

Fig. 1. System model for multiple secondary relay nodes.

Denote $h_{s p}$ and $h_{j p}$ as the channel gain from $\mathrm{SS}$ and $\mathrm{SR}_{j}$ to PBS, respectively. Further assume that each relay knows the channel state information (CSI) of himself, i.e., $h_{s j}$ and $h_{j d}$, and SD node knows all the CSI, i.e., $h_{s 1}, \ldots, h_{s N}$ and $h_{1 d}, \ldots, h_{N d}$ and $h_{1 p}, \ldots, h_{N p}$. We assume that the transmit power of SS and $\mathrm{SR}_{j}$, denoted by $P_{s}$ and $P_{j}$, respectively.

Each transmission period consists of two phases. In the first phase, the SS broadcasts the information $\sqrt{P_{s}} x$, in which $x$ is selected randomly from a binary phase shift keying (BPSK) codebook. Use $|x|$ to denote the magnitude of binary code $x$, then if we normalize $|x|$ to 1 , we have $|x|^{2}=1$ and that average power of the signal emitted by the SS is $P_{s}$. The received signal at $\mathrm{SR}_{j}$ writes

$$
y_{j}=h_{s j} \sqrt{P_{s}} x+n_{j},
$$

where $n_{j}$ represents the additive white Gaussian noise (AWGN) at $\mathrm{SR}_{j}$. During the second phase, $\mathrm{SR}_{j}$ first scales $y_{j}$ as follows,

$$
y_{j} \Rightarrow x_{j}=y_{j} G_{j}
$$

where

$$
G_{j}=\frac{\sqrt{P_{j}}}{\sqrt{1+\left|h_{s j}\right|^{2} P_{s}}}
$$

is the scaling factor, $x_{j}$ is the scaled signal. Then, all SR nodes forward scaled signals to SD simultaneously by sharing the same spectrum with PBS in underlay mode. Here, we denote coefficient $\alpha_{j}$ as the power percentage that $\mathrm{SR}_{j}$ uses to take part in the transmission. We assume SUs can not adaptively adjust their power, then $\alpha_{j}$ can only take the value of 1 or 0 , thus, the relay selection problem is equivalent to the power control problem, i.e., $\alpha_{j}=1$ if $\mathrm{SR}_{j}$ is selected as the relay, or $\alpha_{j}=0$ otherwise.

$\mathrm{SD}$ combines received signals from $\mathrm{SR}_{1}, \cdots, \mathrm{SR}_{N}$ as fol- lows

$$
\begin{aligned}
y= & \sum_{j=1}^{N} \alpha_{j} h_{j d} x_{j}+n \\
= & \sqrt{P_{s}} \sum_{j=1}^{N} \alpha_{j} \frac{\left|h_{s j} h_{j d}\right| \sqrt{P_{j}}}{\sqrt{1+\left|h_{s j}\right|^{2} P_{s}}} x \\
& +\sum_{j=1}^{N} \alpha_{j} \frac{\left|h_{j d}\right| \sqrt{P_{j}}}{\sqrt{1+\left|h_{s j}\right|^{2} P_{s}}} n_{j}+n
\end{aligned}
$$

where $n_{j}$ and $n$ are the i.i.d. complex additive circularly symmetric white Gaussian noise received at $\mathrm{SR}_{j}$ and $\mathrm{SD}$, respectively, with zero-mean and unit-variance.

\section{B. Problem Formulation}

Let $\boldsymbol{\alpha}=\left\{\alpha_{1}, \ldots, \alpha_{N}\right\}$ denotes the relay selection vector, we formulate the problem of the multiple relay selection problem as determining the optimal $\boldsymbol{\alpha}$ which maximizes the signal to interference plus noise ratio (SINR) perceived at SD node subject to the constraint that the IPC of the PBS is guaranteed.

Here, we assume that PBS works at a constant power. Thus, the interference power from PBS to SD is also constant, and maximizing the SINR at SD is equivalent to maximizing the corresponding SNR. Recall that every relay node works in the AF mode, the generalized SNR achieved through maximal ratio combining at $\mathrm{SD}$ writes

$$
\begin{aligned}
S N R_{\text {general }} & =S N R_{\{1, \cdots, N\}} \\
& =\frac{P_{s}\left(\sum_{j=1}^{N} \alpha_{j} \frac{\left|h_{s j} h_{j d}\right| \sqrt{P_{j}}}{\sqrt{1+\left|h_{s j}\right|^{2} P_{s}}}\right)^{2}}{1+\sum_{j=1}^{N} \alpha_{j}^{2} \frac{\left|h_{j d}\right|^{2} P_{j}}{1+\left|h_{s j}\right|^{2} P_{s}}} .
\end{aligned}
$$

The multiple relay selection problem can be formulated as

$$
\begin{aligned}
\boldsymbol{\alpha}^{*}=\arg \max _{\boldsymbol{\alpha}} & S N R_{\text {general }}, \\
\text { s.t. } & \alpha_{j} \in\{0,1\}, \\
& P_{s}\left|h_{s p}\right|^{2} \leq I_{p}, \\
& \sum_{j=1}^{N} \alpha_{j}^{2} P_{j}\left|h_{j p}\right|^{2} \leq I_{p},
\end{aligned}
$$

where $I_{p}$ denotes the IPC of PBS. Constraints in (8) and (9) are imposed by the IPC of the PBS at the two transmission phases, respectively. Recalling that SUs can not adjust their power adaptively, from (8), for every channel reality $h_{s p}$, if $P_{s}>\frac{I_{p}}{\left|h_{s p}\right|^{2}}$, the transmission between SS and SD can not be established.

\section{The Multiple Relay Selection Schemes}

We will next discuss the multiple relay selection schemes in cognitive relay networks. Additionally, as preliminary information, we refer readers to [7] and [12] for the details of knapsack problem and corresponding greedy scheme. 


\section{A. The Greedy Scheme for Multiple Relay Selection}

The multiple relay selection formulated in (6) to (9) is a knapsack-like problem and is also NP-complete. In a cognitive relay network with $N$ SRs, since each relay can be or not be selected, the exhaustive search will result in the exponential $N$ computational complexity and need $N$ feedback bits to differentiate the selected relay nodes. To reduce the complexity, we first adopt the typical greedy algorithm in the knapsack problem to accommodate our relay selection problem.

In greedy algorithm, a relay efficiency function $f_{j}=\frac{S N R_{j}}{I_{r_{j}}}$ is defined for relay node $j$ with $j=1, \cdots, N$, where

$$
S N R_{j}=\frac{P_{s} P_{j}\left|h_{s j} h_{j d}\right|^{2}}{1+P_{s}\left|h_{s j}\right|^{2}+P_{j}\left|h_{j d}\right|^{2}}
$$

and

$$
I_{r_{j}}=P_{j}\left|h_{j p}\right|^{2} \text {. }
$$

$S N R_{j}$ in (10) denotes the SNR perceived at SD when only $\mathrm{SR}_{j}$ works as the relay node, and $I_{r_{j}}$ in (11) represents the interference power from $\mathrm{SR}_{j}$ to the PBS. Here, $S N R_{j}$ and $I_{r_{j}}$ correspond to the profit function and the weight function in the knapsack problem, respectively. Then the greedy algorithm can be described as follows.

1) Sort the $N$ relay nodes in the descending order of efficiency functin $f\left(h_{s j}, h_{j d}, h_{j p}, P_{j}, P_{s}\right)$. Denote the set of the ordered nodes with $\mathcal{S}$.

2) Go through the set $\mathcal{S}$ to select the nodes one by one until exceeding the IPC or arriving the end of the $\mathcal{S}$.

3) The SD broadcasts a value $f_{\text {th }}$ with $f_{\text {th }} \in\left(f_{k+1}, f_{k}\right]$ and $k$ denotes the index of last selected relay.

4) The $\mathrm{SR}_{j}$ compares its $f_{j}$ with $f_{\mathrm{th}}$. If $f_{j} \geq f_{\mathrm{th}}$, the $\mathrm{SR}_{j}$ will cooperate with its full power, or keep silent during this transmission period otherwise.

In the greedy scheme, the amount of feedback bits is $\min \{M, N\}$. Here, we use $M$ bits to represent the real number $f_{\text {th. }}$. So, when the network scale is not too large, e.g., $N \leq M$, the SD will broadcast the $N$ feedback bits. We can see that the greedy scheme has very low complexity and requires small number of feedback bits.

It is worth to mention that our relay selection problem is not an exact knapsack problem, because the SNR functions of different relay nodes are coupled with each other. Hence in the above greedy algorithm the newly added relay node might play a deconstructive role in the SNR function, resulting in some performance degradation. This will be further verified through the numerical results in Section IV.

\section{B. The Hybrid Scheme for Multiple Relay Selection}

Here, we describe the best single scheme (cf. [6]) and take its performance as a reference in Section IV. In the best single scheme, the single node that can result in the greatest SNR and feasible interference to PBS is selected as the relay node, where SNR and interference to PBS of $\mathrm{SR}_{j}$ are same with (10) and (11). This scheme gets the optimal performance when only one node can be selected as the relay.

Due to the fact that its efficiency function is not the optimal ordering function, the greedy scheme does not necessarily get
TABLE I

\begin{tabular}{|c|c|c|c|}
\hline scheme & $A^{*}$ & times of sort & feedback bits \\
\hline exhaustive & $O\left(2^{N}\right)$ & 1 & $\bar{N}$ \\
\hline best single & 0 & 1 & $\log _{2} N$ \\
\hline greedy & 0 & 1 & $\min \{M, N\}$ \\
\hline hybrid & $O(N)$ & 2 & $1+T^{*}$ \\
\hline
\end{tabular}

COMPleXITy COMPARISON AMONG DIFFERENT SCHEMES

the optimal performance. To overcome the drawback of the greedy algorithm, we propose a hybrid scheme, which implies a combination of the best single scheme and the evolved greedy scheme.

Based on the efficiency function defined in Section III-A and the greedy algorithm, the hybrid scheme is described as follows.

- If only one relay node is selected in the greedy algorithm, the hybrid scheme employs the best single scheme.

- If multiple relay nodes can be selected in the greedy algorithm, the hybrid scheme chooses the $k$ such that $S N R_{\{1 \ldots k\}}$ is the largest among $\left\{S N R_{\{1\}}, \cdots, S N R_{\{1 \cdots k\}}, \cdots, S N R_{\{1 \ldots N\}}\right\}$ subject to the constraint that the IPC is guaranteed. It is worthy pointing out that value of the optimal $k$ might be different from the relay node number selected by the greedy algorithm.

- The SD uses simple encoding technology to differentiate the results. When only one node is selected, the SD broadcasts " $0+T$ " with $T=\log _{2} N$ denoting the index of the selected relay node. When multiple relay nodes are selected, the SD broadcasts " $1+T$ " with $T=\min \{M, N\}$ denoting the threshold value $f$ th or the $N$ bits.

As above mentioned, the hybrid scheme judiciously combines the best single scheme and the evolved greedy scheme. So it is expected that the hybrid scheme can guarantee the performance no worse than that achieved by the best single scheme or the greedy scheme. Later in this paper it is shown that the hybrid scheme can achieve the near-optimal performance.

\section{Complexity Analysis of Different Schemes}

Complexity and the feedback bits are summarized in Table I. We can see that the exhaustive scheme has the maximum complexity, while both the greedy scheme and the best single scheme have the similar complexity, except that the number of selected node is different. There is always a tradeoff between the performance and the complexity: improving the performance will require more computational complexity and the amount of feedback bits. The hybrid scheme achieves a better tradeoff than other schemes. This will be further verified through the numerical results in the following section IV.

\section{Simulation Results}

In this section, simulation results are presented for performance evaluation. For simplicity, we assume that all SUs have the same transmit power of $P_{s}$, i.e., $P_{n}=P_{s}, n=1, \cdots, N$, 

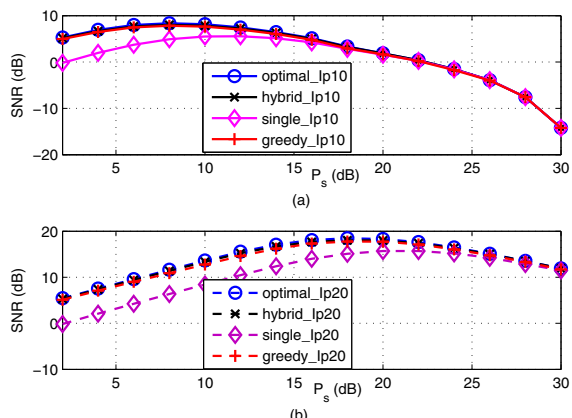

Fig. 2. Average SNR performance of four schemes, exhaustive search, greedy, hybrid and best single with $I_{p}$ of $20 \mathrm{~dB}$ (a) and $10 \mathrm{~dB}$ (b).

and that all channels experience the i.i.d. Rayleigh fading. All results are obtained by averaging over 10000 simulations.

Fig. 2 shows the perceived SNR with five potential relay nodes and in the cases of $I_{p}=20 \mathrm{~dB}$ and $I_{p}=10 \mathrm{~dB}$, respectively. Fig. 3 gives the corresponding number of the selected relay nodes. From Fig. 2 we can see that, the SNR increases as the transmit power increases in the low and moderate power areas (e.g., below $18 \mathrm{~dB}$ when $I_{p}=20 \mathrm{~dB}$ ), but decreases at high power area. This is caused by the following factors. First, if the transmit power of $P_{s}$ exceeds a threshold, which is $\frac{I_{p}}{\left|h_{s p}\right|^{2}}$ in our case, there would be no transmission link established between SS and SD. Second, as the transmit power increases, the interference from SRs to the PU will also increase and hence the number of selected relay nodes will be reduced. This can be verified by Fig. 3, from which we can also see that the IPC can considerably affect the SNR performance and that higher IPC would lead to better SNR performance as the transmit power increases.

Fig. 4 shows the SNR performances in cases of 11 and 15 potential relay nodes, respectively. We can see that the proposed hybrid scheme always outperforms the greedy and single schemes and can achieve nearly optimal performance in SNR. It is interesting to notice that although the greedy scheme sometimes selects the most relay nodes, its SNR performance has never been the best. This implies that the efficiency function used in greedy scheme is not the most efficient in terms of ordering the relay nodes whereas the proposed hybrid scheme can overcome this drawback. In other words, the traditional greedy scheme is not applicable to this kind of knapsack-like problem, in which multiple interfering items are coupled with each other, while the proposed hybrid scheme is more desirable.

\section{CONCLUSION}

In this paper we have studied the problem of multiple relay selection in cognitive relay networks. A hybrid scheme has been proposed and the greedy and single-best schemes have been investigated for performance comparison. Performance of different schemes has been evaluated in simulation results. It has been shown that the conventional greedy scheme is not always suitable to multiple relay selection, as multiple relay nodes are coupled with each other in terms of SNR expression. The proposed hybrid scheme can overcome the drawback of the greedy scheme and can achieve near optimal
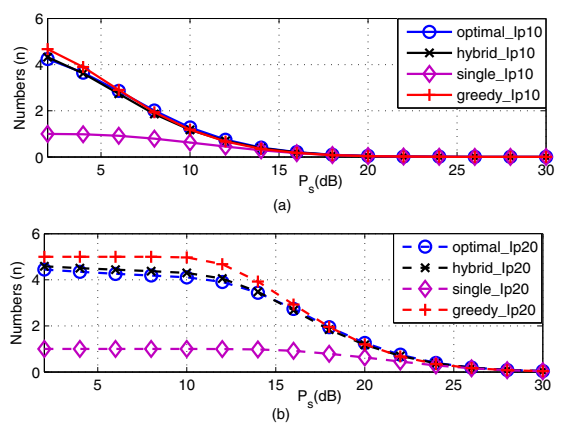

Fig. 3. Average number of the selected relay nodes in exhaustive search, greedy, hybrid and best single schemes with $I_{p}$ of $20 \mathrm{~dB}$ (a) and $10 \mathrm{~dB}$ (b).
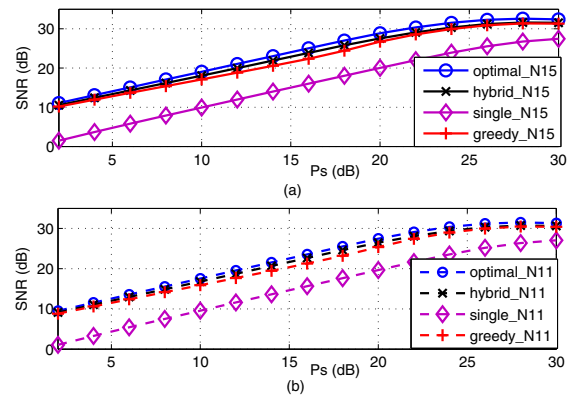

Fig. 4. Average SNR performance of the four schemes, exhaustive search, greedy, hybrid and best single in cases of 11 relay nodes(a) and 15 relay nodes (b) with $I p=30 \mathrm{~dB}$.

SNR performance. Complexity and the required feedback bits in the proposed scheme have also been analyzed.

\section{REFERENCES}

[1] C.-X. Wang, H.-H. Chen, X. Hong, and M. Guizani, "Cognitive radio network management", IEEE Vehi. Techno. Magazine, vol. 3, no. 1, pp. 28-35, Mar. 2008.

[2] A. Nosratinia, T.E. Hunter, A. Hedayat, "Cooperative Communication in Wireless Networks,” IEEE Commun. Magazine, Oct. 2004.

[3] Q. Zhang, J. jia and J. Zhang, "Cooperative Relay to Improve Diversity in Cognitive Radio Networks," IEEE Commun. Magazine, Feb. 2009.

[4] C.-X. Wang, X. Hong, H.-H. Chen, and J. S. Thompson, "On capacity of cognitive radio networks with average interference power constraints", IEEE Trans. Wireless Commun., vol. 8, no. 4, pp. 1620-1625, Apr. 2009.

[5] X. Hong, C.-X. Wang, M. Uysal, X. Ge, and S. Ouyang "Capacity analysis of hybrid cognitive radio networks with distributed VAAs," IEEE Trans. Vehi. Techno., vol. 59, no. 7, pp. 3510-3523, Sept. 2010.

[6] Y. Jing, H. Jafarkhani, "Single and Multiple Relay Selection Schemes and their Achievable Diversity Orders," IEEE Trans. on Wireless Commun., vol. 8, no. 3, pp. 1414-1423, March 2009.

[7] D.S. Michalopoulos, G. K. Karagiannidis, T. A. Tsiftsis, and R. K.Mallik, "An optimized user selection method for cooperative diversity systems," in Proc. IEEE Globecom'06, Nov.-Dec. 2006.

[8] K. Lee and A. Yener, "Outage performance of cognitive wireless relay networks," in Proc. IEEE Globecom'06, Nov. 2006.

[9] X. Gong, W. Yuan et al., "A Cooperative Relay Scheme for Secondary Communication in Cognitive Radio Networks," in Proc. of IEEE Globecom'08, Nov. 2008.

[10] C. Sun, B. Letaief, "Uer Cooperation in Heterogeneous Cognitive Radio Networks with Interference Reduction," in Proc. of IEEE ICC'08, May 2008.

[11] J. Mietzner, L. Lampe, R. Schober, "Distributed Transmit Power Allocation for Relay-Assisted Cognitive-Radio Systems," in Proc. of IEEE ACSSC 2007, Nov. 2007.

[12] H. Kellerer, U. Pferschy, and D. Pisinger, Knapsack Problems, 1st ed. Berlin: Springer, 2004.

[13] A. Goldsmith, Wireless Communication, Cambridge University Press, 2005. 